\section{Management of day- surgery patients with cataract attending a peripheral ophthalmic clinic}

KAREN ROSE, HEATHER WATERMAN, LINDA TOON, DAVID MCLEOD,

ANDREW TULLO

\section{Abstract}

Purpose To compare two organisational models of management for patients with cataract referred to a peripheral ophthalmic clinic who underwent day-surgery at a main eye hospital.

Method Patients were randomised into two groups. The experimental group ( $n=25)$ received pre-operative assessment by a trained ophthalmic nurse at the peripheral clinic immediately following diagnosis of cataract and diary-booking for surgery. The control group $(n=24)$ received a separate appointment for pre-operative assessment at the main hospital. For all patients, the first review appointment ( 3 or 5 days post-operatively) and all subsequent review was at the peripheral clinic. Outcome measures included: visual acuity, subjective visual function (VF-14), anxiety and depression (HADS), semistructured interviews to ascertain patient satisfaction, and a cost-benefit analysis.

Results There were no significant differences at any time between the experimental and control groups with respect to visual acuity, subjective visual function or anxiety and depression. The experimental model was found to be more cost-effective and provided a less fragmented means of care delivery. The majority of patients in both groups expressed satisfaction with their care but, overall, the experimental model was preferred.

Conclusions Nurse-led pre-operative assessment of patients with cataract at a peripheral ophthalmic clinic is safe, cost-effective and is preferred by patients.

Key words Cataract, Cost-benefit analysis, Day-surgery, Ophthalmic out-patients, Pre-operative assessment, Randomised controlled trial
Day-surgery for cataract is safe $\mathrm{e}^{1-6}$ and costeffective ${ }^{1,2,7-10}$ and is becoming the preferred means of managing a condition that is the most common reason for referral to ophthalmic outpatient departments. ${ }^{11}$ The Audit Commission, reporting in $1990,{ }^{12}$ suggested that $20 \%$ of cataract surgery should be performed on a daycare basis; since then, more ambitious targets of at least $80 \%$ have been set. ${ }^{12,13}$

The change from in-patient to day-surgical care has organisational implications, yet little research has been carried out into this aspect of management. Two exceptions are studies carried out in Bristol and Oxford. The Bristol study, comparing outcomes for in-patient and day-surgery, included not only clinical outcome measures but also patient satisfaction and preference, $^{14-16}$ and also evaluated an enhanced role for an ophthalmic nurse. ${ }^{17}$ The study showed that day-surgery for cataract was a suitable option for the majority of patients provided both medical and social factors were taken into account. The Oxford study, ${ }^{18}$ assessing how resources might best be utilised in relation to pre-operative assessment, concluded that a separate pre-operative assessment conferred negligible benefit.

The need for more studies that consider the organisational implications of day-surgery for cataract seems clear. Many changes in practice are made on an ad hoc basis rather than on research-based evidence. While, at times, a proposed change may seem self-evidently for the better, an evidence-based culture should not allow such assumptions to be freely made.

We were concerned how best to organise day-surgery for patients referred to a peripheral ophthalmic clinic who subsequently received their surgery at a main eye hospital. Postal surveys (Appendices A and B) showed that such an investigation was likely to be relevant to a large number of ophthalmology departments. ${ }^{19}$ A randomised, controlled study was designed to ascertain the preferred site for pre-operative assessment (peripheral clinic or main hospital) and to assess the value of
K. Rose

H. Waterman

School of Nursing,

Midwifery and Health

Visiting

Coupland III

University of Manchester

Manchester, UK

K. Rose

H. Waterman

D. McLeod

A. Tullo

University Department of Ophthalmology

Manchester Royal Eye

Hospital

Manchester, UK

L. Toon

Health Services

Management Unit

University Precinct

Manchester, UK

Dr K. Rose

University Department of

Ophthalmology

Manchester Royal Eye

Hospital

Oxford Road

Manchester M1 $39 \mathrm{WH}$, UK

Tel: +44 (0)1612765522

This study was funded by the Department of Health Primary/Secondary Care Interface Research Initiative

Received: 11 August 1998 Accepted: 23 October 1998 
introducing a trained ophthalmic nurse into the care of patients with cataract attending the peripheral clinic. Approval to carry out the study was obtained from the local Research Ethics Committee.

\section{Patients and methods}

Patients with cataract attending a peripheral ophthalmic clinic some $5 \mathrm{~km}$ distant from the main hospital were diary-booked for surgery at time of diagnosis and were randomly drawn to receive either an experimental (group A) or control (group B) model of care. Table 1 outlines the model of care for both groups. The experimental model differed in that patients received immediate pre-operative assessment by a trained ophthalmic nurse, thereby eliminating an entire appointment at the main hospital.

Pre-operative assessment at both sites included: recording Snellen visual acuity, biometry, keratometry, general health check (pulse, blood pressure, urine test), nursing history (details of social circumstances, medication, etc.), and the giving of information to patients about surgery.

At the peripheral hospital, after confirmation of their diagnosis by the ophthalmologist, group A patients were seen by a trained ophthalmic nurse who carried out all aspects of pre-operative assessment in one episode apart from obtaining consent for surgery, which was done by medical staff on the day of surgery. Patients in group $B$, who made an extra visit to the main hospital for pre-operative assessment, had up to four separate points of staff contact with different staff groups.

All patients were operated on at the main hospital on a Wednesday or Friday. All subsequent routine followup took place at the peripheral hospital on a Monday. Following the recommendations of Tufail et al., ${ }^{20}$ it was decided that a first post-operative review at up to 7 days was acceptable practice. Patients were therefore seen 3 or 5 days post-operatively (unless the surgeon felt a firstday post-operative assessment was necessary).

Apart from Snellen visual acuity, all study data were collected by an independent researcher. Questionnaires to measure subjective visual function (VF-14 ${ }^{21}$ ), and anxiety and depression (Hospital Anxiety and Depression Scale, $\mathrm{HADS}^{22}$ ) were administered; these were analysed using a repeated measures analysis of variance. Semi-structured interviews were undertaken to ascertain patient satisfaction with care, and interview and observational data were recorded relating to costbenefit analysis. The VF-14 was administered at the first clinic visit, the first post-operative follow-up and 3 months post-operatively in the patients' homes. The HADS was administered at the first clinic visit, postoperatively on the day of surgery and at the 3 month home visit. Cost data were collected at the first clinic visit, on the day of operation and at the first follow-up for all patients and for group B patients at pre-operative assessment. Interview data were recorded at all researcher/patient interactions. It was felt to be important to conduct the final phase of data collection in patients' homes in order to maximise the potential for the interview to be a social interaction ${ }^{23}$ (as opposed to a clinical adjunct) where patients would feel more able to voice any criticisms of the system.

Over a 12 month period commencing in September 1995, 58 patients (each with age-related cataract and awaiting surgery to their first eye), who were suitable for day-surgery under local anaesthetic and who had no major ocular co-morbidities, gave informed consent to take part in the study.

\section{Results}

\section{Completion and complications}

A total of 49 patients completed the study. In group A, 18 women and 7 men (mean age 77 years) completed. Three patients failed to complete: 2 withdrew without giving a reason and 1 had short-term memory loss. The mean waiting times from general practitioner (GP) referral to first hospital visit and from then to surgery were 3.5 months and 3 months respectively. Sixteen patients underwent phacoemulsification, 2 of whom had intraoperative complications (zonular dehiscence and dropped nucleus). Nine patients underwent extracapsular cataract extraction (ECCE), 1 of which was a conversion from phacoemulsification. Two of the 9 had post-operative complications (toxic reaction to sutures and post-operative inflammation). Eight of the 25 operations were performed by the consultant, 12 by a registrar and 5 by a senior house officer.

Table 1. Comparative management plans for day-surgery cataract patients

\begin{tabular}{|c|c|}
\hline Group A & Group B \\
\hline \multicolumn{2}{|c|}{ Referral by GP } \\
\hline Peripheral clinic attendance & Peripheral clinic attendance \\
\hline Cataract confirmed & Cataract confirmed \\
\hline Diary-booked for surgery & Diary-booked for surgery \\
\hline \multirow[t]{5}{*}{ Pre-operatively assessed } & Diary-booked for pre-operative assessment at main hospital \\
\hline & Pre-operative assessment ( $2-4$ weeks before surgery) \\
\hline & Surgery at main hospital \\
\hline & First post-operative visit at peripheral clinic ( $3-5$ days post-op) \\
\hline & Subsequent follow-up at peripheral clinic \\
\hline
\end{tabular}


In group B, 18 women and 6 men (mean age 76 years) completed. Six patients failed to complete: 2 had an intercurrent illness, 1 declined surgery, 1 did not attend for pre-operative assessment, 1 was lost to follow-up and 1 withdrew without giving a reason. The mean waiting times from GP referral to first hospital visit and from then to surgery were 4 months and 3 months respectively. Sixteen patients underwent phacoemulsification with 1 intraoperative complication (capsular rupture with vitreous loss) and 2 postoperative complications (iritis and a possible allergic reaction to neomycin drops). Eight patients underwent ECCE, 1 of whom had a possible allergic reaction to neomycin. Six of the 24 operations were performed by the consultant, 14 by a registrar and 4 by a senior house officer.

Power calculations by a medical statistician showed that the sample size was sufficient to detect a difference between groups of the order of $1 \mathrm{SD}$ of a continuous measure at the $5 \%$ level.

\section{Change in visual acuity}

Records of pre-operative visual acuity were available for 48 of the patients. In both groups, patients undergoing ECCE were more likely to have a pre-operative visual acuity of $6 / 36$ or worse compared with

phacoemulsification ( $94 \%$ compared with $40 \%$ ) and $88 \%$ of ECCE patients achieved a final visual acuity of $6 / 12$ or better compared with $97 \%$ of patients undergoing phacoemulsification. These results were comparable for the two groups.

\section{Subjective visual function}

The score range for the VF-14 questionnaire is 0-100; a score of 100 indicates no problems with performing any applicable task while 0 indicates inability to perform any applicable task. The mean VF-14 scores for each group at the three times of administration are shown in Table 2.

Table 2. Mean subjective visual function scores using VF-14 and mean depression and anxiety scores using HADS

\begin{tabular}{lrr}
\hline & Group A & Group B \\
\hline Time 1 & & \\
VF-14 & 68.148 & 58.204 \\
Depression & 5.520 & 5.750 \\
Anxiety & 8.000 & 7.667 \\
Time 2 & & \\
(a) VF-14 & 87.060 & 85.696 \\
(b) Depression & 5.120 & 4.708 \\
(b) Anxiety & 8.000 & 7.042 \\
Time 3 & & \\
VF-14 & 95.896 & 94.108 \\
Depression & 4.120 & 4.500 \\
Anxiety & 6.360 & 5.667 \\
\hline
\end{tabular}

Time 1, first clinic visit; time 2 (a), first post-operative visit; time 2 (b), day of operation; time 3 , home visit (3 months postoperatively).
There were no overall differences between the two groups $(p=0.119)$ at the three time points tested and also no significant interaction with time $(p=0.141)$; in other words, groups A and B behaved in the same way over the time points. To clarify this, the time points 1 and 2, and 2 and 3 were compared separately. There were no group-related differences but highly significant differences were evident within each group between both times 1 and $2(p=0.001)$ and times 2 and 3 $(p=0.007)$, indicating a marked improvement in subjective visual function over time.

\section{Depression and anxiety}

For each component of HADS the score range is $0-21$, 0 indicating no evidence of depression or anxiety and 21 indicating extreme depression or anxiety. Table 2 shows the mean depression and anxiety scores for each group. Each component was analysed separately.

Analysis of the depression component showed that there were no statistically significant differences between the groups at times 1,2 and $3(p=0.939)$ but a significant reduction in level of depression over time for both groups $(p=0.01)$. The groups showed no statistically significant difference in behaviour $(p=0.614)$ over times 1,2 and 3.

Analysis of the anxiety component showed that there were no statistically significant differences between the groups at the three time points tested $(p=0.526)$ but a significant difference in level of anxiety over time for both groups $(p=0.001)$. In addition, the groups showed no statistically significant difference in behaviour over the three time points $(p=0.81)$.

The fact that both groups showed significant improvement in levels of both depression and anxiety post-operatively indicates that patients experienced an improvement in sense of well-being after surgery, regardless of group.

\section{Cost-benefit analysis}

The cost-benefit analysis considered costs to both patients and the National Health Service (NHS). To assess these, data were collected on the cost of transport and staff, time spent at pre-operative assessment, and implications for patients and carers. These were comparable for both groups with the exception of costs incurred for pre-operative assessment.

Group B patients made an extra visit to the main ophthalmic hospital for pre-operative assessment. Of the 24 patients in group B, 22 arranged their own transport to this appointment (private car, taxi or public transport), resulting in a mean additional cost of $£ 4.82$. Two patients came by hospital transport, resulting in a mean additional cost to the NHS of $£ 26.30$.

There were other costs involved for group B. Fifteen of these patients were accompanied by carers, 4 of whom had to take time away from work and a further 2 of whom had to arrange child care. In addition, as well as making an extra hospital visit, group B patients also 
Table 3. Time (in minutes) to undergo pre-operative assessment

\begin{tabular}{lcc}
\hline & Group A & Group B \\
\hline Unqualified nursing time & - & 6 \\
Mean & - & $4-11$ \\
Range & 44 & \\
Qualified nursing time & $28-84$ & 34 \\
Mean & & $18-58$ \\
Range & - & \\
Medical time & - & 13 \\
Mean & & $5-26$ \\
Range & 11 & \\
Waiting time & $0-36$ & $18-189$ \\
Mean & & \\
Range & 55 & $60-259$ \\
Total clinic time & $33-94$ & \\
Mean & & \\
Range &
\end{tabular}

spent much longer in pre-operative assessment. Comparative times for groups A and B are shown in Table 3. This, in turn, led to an increased cost to the NHS. The block cost of an out-patient appointment quoted by both the peripheral and the main hospital for the financial year 1996/7 (the main financial year of the study) was $£ 45.00$. This was not broken down into staffing or overheads costs. However, it was possible to use the data gathered by the researcher at pre-operative assessment to produce a comparative estimate of the staffing costs for pre-operative assessment in the two models (Table 4).

\section{Patient satisfaction}

At interview, patients generally expressed high levels of satisfaction with their care, focusing on the visual outcome. However, there was agreement that the more care that was available at the peripheral hospital, the better. This preference centred on the following factors: the peripheral hospital was more easily accessible; patients were more familiar with the peripheral site; waiting and travelling times (especially in relation to preoperative assessment) were shorter at the peripheral clinic; and transport to the peripheral clinic was easier to organise and less expensive. These factors meant that patients felt they had to rely less on carers.

\section{Discussion}

There was no difference in final visual acuity between patients in group A and group B, the only differences relating to type of operation (phacoemulsification or

Table 4. Comparative staff costs for pre-operative assessment

\begin{tabular}{lcc}
\hline & Group A & Group B \\
\hline Minimum cost & $£ 22.92$ & $£ 23.46$ \\
Maximum cost & $£ 32.44$ & $£ 39.59$ \\
Mean cost & $£ 25.64$ & $£ 29.28$ \\
\hline
\end{tabular}

ECCE). Acuity in both groups compared favourably with the National Cataract Surgery Survey, ${ }^{3}$ with over $90 \%$ achieving a visual acuity of $6 / 12$ or better, thus indicating that both the control and experimental models of organisational management are compatible with a good visual outcome. The results from the VF-14, relating to subjective visual function, support this conclusion. Patients in both groups experienced a highly significant improvement in their ability to perform everyday tasks. Thus, the two groups were comparable at the time points tested and behaved (improved) in the same way. Similarly, differences in the organisational models of management did not affect patients' psychological wellbeing, which uniformly improved after surgery.

The cost-benefit analysis demonstrated that there was some financial advantage to the experimental model, for both patients and the NHS. Transport costs to the peripheral hospital, which was located nearer to patients' homes, were obviously lower. In addition, the entire cost of a visit to the main hospital is saved if pre-operative assessment is carried out immediately following diagnosis at the peripheral clinic. In a group of elderly and often infirm patients, it is also kinder to reduce the amount of travelling they have to do. It is not possible to draw definitive conclusions about transport costs from our relatively small sample. Nevertheless, we estimate that one saved pre-operative visit in the management of patients with cataract from peripheral clinics would result in the avoidance of tens of thousands of journeys per annum nationwide and hundreds of thousands of patientmiles travelled (Appendices A and B).

There was also a time cost difference between groups. Group B patients could expect, on average, to spend 2.5 times as long in pre-operative assessment as group A patients. Of this, less than $40 \%$ was spent receiving care from staff and more than $60 \%$ was waiting time. In contrast, the much shorter time that group A patients spent in pre-operative assessment broke down to $20 \%$ of time spent waiting and $80 \%$ of time spent receiving care. In addition, group $\mathrm{A}$ patients received more individualised, less task-oriented care with all elements of their assessment being undertaken in one seamless interaction with the ophthalmic nurse. This effect was enhanced by the fact that the nurse at the peripheral clinic also (usually) worked at the main hospital and therefore provided an important continuity link for patients. This was helpful both administratively and because she would often be available to patients on the day of their surgery:

The value of the experimental model is further enhanced by the fact that reducing the number of hospital attendances consequentially reduces the number of times patients need someone to accompany them, the potential disruption this can cause and the sense of dependence and 'being a nuisance' that this can create for patients. As the interview data showed, patients' preference was clearly for the peripheral clinic and a reduced number of hospital appointments. 


\section{Summary}

Day-surgery for cataract, while safe and cost-effective, has implications for patients and their families, placing a greater burden of care and responsibility on them. It is therefore important to ensure that the organisation of their treatment is directed towards minimising the difficulties they encounter. In this study of patients with cataract referred to a peripheral ophthalmic clinic, we conclude that the preferred model of care is the experimental model and this is consequently now in routine use.

\section{Appendix A. Peripheral clinics based on eleven ophthalmic units in the North West Region Survey by Tullo and Rose, 1996/7.}

No. of peripheral out-patient clinic sessions per week based on each main hospital: 1-11 Minimum distance (main to peripheral hospital): $3-48 \mathrm{~km}$ Maximum distance (main to peripheral hospital): 10-64 $\mathrm{km}$

Site of pre-operative assessment: 2 peripheral, 9 main Minimum no. of days to first post-operative assessment: 0-14 (0 = same day)

Site of first post-operative assessment: 5 peripheral, 2 home visits, 4 main

\section{Appendix B. Peripheral clinics based on ten large provincial regional teaching ophthalmic units in England}

Survey by Tullo and Rose, 1996/7.

No. of peripheral out-patient clinic sessions per week based on each main hospital: 1-10

Minimum distance (main to peripheral hospital): $5-38 \mathrm{~km}$

Maximum distance (main to peripheral hospital): 20-112 $\mathrm{km}$

Site of pre-operative assessment: 1 peripheral, 9 main Minimum no. of days to first post-operative assessment: $1-7$

Site of first post-operative assessment: 2 peripheral, 7 main, 1 no response

The authors would like to thank Dr V. Hillier, Department of Medical Biophysics, University of Manchester, for her assistance with the statistical analysis of the data and Mr S. Charles, Consultant Ophthalmic Surgeon, Manchester Royal Eye Hospital, for his comments on the draft of this paper.

\section{References}

1. Aylward G, Larkin, D, Cooling R. Audit of cost and clinical outcome of cataract surgery. Health Trends 1993;25:126-9.
2. NHS Centre for Reviews and Dissemination. Effective health care bulletin: management of cataract. Edinburgh: University of York, NHS Centre for Reviews and Dissemination/ Churchill Livingstone, 1996.

3. Desai P. The national cataract surgery survey. II. Clinical outcomes. Eye 1993;7:489-94.

4. Ingram RM, Bannerjee D, Traynar MJ, Thompson RK. Daycase cataract surgery. Br J Ophthalmol 1983;67:278-81.

5. Javitt JC, Street DA, Tielsch JM, Wang Q, Kolb MM, Schein O, et al. (on behalf of the Cataract Patients Outcomes Research Team). National outcomes of cataract extraction: retinal detachment and endophthalmitis after outpatient cataract surgery. Ophthalmology 1994;101:100-5.

6. Strong NP, Wigmore W, Smithson S, Rhodes R, Woodruff G, Rosenthal AR. Daycase cataract surgery. Br J Ophthalmol 1991;75:731-3.

7. Cooper JM. Day case cataract surgery in the UK and USA: a comparative study. Br J Nurs 1997;6:39-43.

8. Davies PD, Limacher E, Powell K. Out-patient cataract surgery 1982-1986. Eye 1987;1:728-34.

9. Drummond MF, Yates JM. Clearing the cataract backlog in a (not so) developing country. Eye 1991;5:481-6.

10. Watts MT, Pearce JL. Daycase cataract surgery. Br J Ophthalmol 1988;72:897-9.

11. Williams MH, Frankel SJ, Nanchalal K, Coast J, Donovan J. Cataract surgery: health care needs assessment - the epidemiologically-based needs assessment reviews. Oxford: Radcliffe Medical Press Ltd, 1994.

12. Audit Commission for Local Authorities and NHS in England and Wales. A short-cut to better services: day surgery in England and Wales. London: HMSO, 1990.

13. NHSME in Scotland/Scottish Health Service Advisory Council. Day case surgery for cataract. Scotland: HMSO, 1992.

14. Lowe KJ, Gregory DA, Jeffery RI, Easty DL. Patient perceptions and social impact: preliminary results of the Bristol MRC study. Eye 1991;5:373-8.

15. Lowe K, Gregory D, Jeffery R, Easty D. Cataract extraction: patient characteristics and preferences. Health Trends 1992:24:151-3.

16. Lowe KJ, Gregory DA, Jeffery RI, Easty DL. Suitability for daycase cataract surgery. Eye 1992;6:506-9.

17. Gregory D, Lowe KJ. An enhanced role for the ophthalmic nurse: a control study on day case cataract. Professional Nurse 1991;7:43-4,46,48.

18. Prasad S, Tanner V, Patel CK, Rosen P. Optimisation of outpatient resource utilisation in cataract management. Eye 1998;12:403-6.

19. Rose KE, Waterman HA, Toon L, McLeod D, Tullo AB. Comanaged care in a peripheral ophthalmic clinic. University of Manchester/Central Manchester Health Care Trust: Unpublished Report for the Department of Health, 1997.

20. Tufail A, Foss AJ, Hamilton AM. Is the first day postoperative review necessary after cataract extraction? $\mathrm{Br} \mathrm{J}$ Ophthalmol 1995;79:646-8.

21. Steinberg EP, Tielsch JM, Schein OD, Javitt JC, Sharkey P, Cassard SD, et al. The VF-14: an index of functional impairment in patients with cataract. Arch Ophthalmol 1994;112:630-7.

22. Zigmond AS, Snaith RP. The hospital anxiety and depression scale. Acta Psychiatr Scand 1983;68:361-70.

23. Hammersley M, Atkinson P. Ethnography: principles in practice. London: Routledge, 1990. 